\title{
Epidemiology: what it is and why it matters
}

\author{
INVITED COMMENTARY ON ... CANNABIS USE AND PSYCHOSIS ${ }^{\dagger}$
}

\author{
Brendan D. Kelly
}

Abstract Epidemiology is the study of why, how and how often diseases occur in given populations. There is now
a sufficiency of epidemiological evidence for psychiatrists to warn that cannabis use could increase risk
of psychosis in later life. The policy impact of this accumulated epidemiological evidence is difficult
to predict. There is a strong need to develop models of mental health policy-making that incorporate
careful interpretation of epidemiological evidence.

Epidemiology is the study of why, how and how often diseases occur in given populations. Epidemiology is essentially concerned with counting: How many people in the population develop the disease of interest? How many of these people were exposed to the risk factor of interest? How many people in the population do not develop the disease? How many of these people were exposed to the risk factor? What does all of this mean?

Macleod (2007, this issue) examines the epidemiological evidence for an association between cannabis use and psychotic symptoms, and explores its implications for policy and practice. His article provides a particularly valuable discussion of key epidemiological and methodological issues relevant to both the interpretation of evidence and the development of health and social policy. Macleod wisely starts with the most fundamental questions of all: Might cannabis use cause psychosis? Might psychosis cause cannabis use? Might an apparent association between the two arise as an artefact of the way in which the relation is studied? Might they share common antecedents? These are the kinds of simple, logical questions that constitute the basis of much epidemiological work and, in turn, assist with the interpretation of evidence resulting from epidemiological studies.

${ }^{+}$See pp. $400-411$, this issue.

\section{Causation and clinical pragmatics}

One of the central aims of epidemiological research is to identify the causes of diseases, and, in this vein, studies of cannabis and psychosis generally aim to characterise the extent to which it is reasonable to regard cannabis as a risk factor for future psychosis. The evidence in favour of such an association is now considerable, as shown by the studies cited by Macleod and the conclusions of other reviews (e.g. Moore et al, 2007). From an epidemiological perspective, it is useful to consider these findings in the context of the Bradford Hill criteria for causation, which include the strength, consistency and specificity of the association, temporal sequence of events, biological gradient, biological rationale, coherence, experimental evidence and analogous evidence (Hill, 1965; Doll, 1992). More detailed considerations of some of these issues in relation to cannabis are provided by Castle \& Murray (2004), Macleod et al (2004) and Moore et al (2007). VanReekum et al (2001) provide a valuable overview of the general relevance of the Bradford Hill criteria in the context of neuropsychiatry.

In addition to its role in identifying causal relationships, epidemiological research also serves another extremely useful function by producing comprehensible, pragmatic findings even when a disease is associated with multiple risk factors, some of which are unknown and others of which are

Brendan D. Kelly is Senior Lecturer in Psychiatry at University College Dublin (Department of Adult Psychiatry, University College Dublin, Mater Misericordiae University Hospital, 62/63 Eccles Street, Dublin 7. Email: brendankelly35@gmail.com). Research interests include the epidemiology of psychosis and relationships between mental illness and social factors. He was joint winner of the Royal College of Psychiatrists' Gaskell medal and prize in 2003. 
incompletely understood. In these circumstances, careful epidemiological consideration of individual risk factors in studies that control, insofar as is practicable, for other risk factors, can still reach useful, pragmatic conclusions: for example the identification of an increased risk of psychosis in individuals who have ever used cannabis, with a pooled adjusted odds ratio of 1.41 (Moore et al, 2007). This means - very simply - that current best evidence indicates that the risk of psychosis for someone who has ever used cannabis is 1.4 times the risk of psychosis for someone who has never used cannabis. This finding is not only statistically and clinically significant, it is also comprehensible.

\section{Policy and practice}

It is a fundamental tenet of epidemiological research that findings such as these should be used, with appropriate care, to shape current clinical practice, future research and ongoing health policy-making. The clinical and policy implications of the association between cannabis and psychosis are both far-reaching and controversial. As Macleod points out, there are already myriad reasons to encourage young people to avoid cannabis use, based on various adverse effects other than increased risk of psychosis. In addition, however, current evidence clearly identifies future psychosis as another substantive risk associated with cannabis and, indeed, indicates that cannabis use may also occur in individuals with established psychosis, further compounding and complicating their illness.

Given the substantial public interest in studies of cannabis use, Macleod's straightforward, logical, rigorous approach to the epidemiological evidence is as valuable as it is rare. His article will undoubtedly prove invaluable for mental health professionals who need to explain the risks to mental health service users, families, carers and at-risk groups (e.g. school populations). In particular, this article will assist mental health service providers who face increasingly detailed questions from well-informed stakeholders, as well as colleagues, policy-makers, service-planners and the media. At the most basic clinical level, there is clearly now a sufficiency of evidence for psychiatrists to inform all of these that cannabis use could increase risk of psychosis in later life (Moore et al, 2007). There is still, however, a need for further study, especially in relation to the availability and effects of different forms and strengths of cannabis. This is a particularly important issue in light of ongoing public concern about the reported increased availability of stronger forms of cannabis in recent years.

The likely policy impact of this accumulated epidemiological evidence is difficult to predict. As Macleod points out, short-term political concerns are a significant factor, as are the competing voices of diverse interest groups who place varying amounts of emphasis on epidemiological findings. There is certainly a strong need for epidemiological findings to inform mental health policy-making (Jenkins et al, 2007) and to provide a coherent link between health policy, drugs policy and social policy. Increasingly, these policy directions will be determined at both national and international levels (Kelly, 2007), further emphasising the importance of careful interpretation of epidemiological evidence and judicious development of policy models that combine the best available evidence with coherent, comprehensible approaches to policy-making.

\section{Declaration of interest}

None.

\section{References}

Castle, D. \& Murray, R. (eds) (2004) Marijuana and Madness: Psychiatry and Neurobiology. Cambridge University Press.

Doll, R. (1992) Sir Austin Bradford Hill and the progress of medical science. BMI, 305, 1521-1526.

Hill, A. B. (1965) The environment and disease: association or causation? Proceedings of the Royal Society of Medicine, 58, 293-300.

Jenkins, R., McDaid, D., Brugha, T., et al (2007) The evidence base in mental health policy and practice. In Mental Health Policy and Practice across Europe (eds M. Knapp, D. McDaid, E. Mossialos, et al), pp. 100-125. McGraw-Hill/Open University Press.

Kelly, B. D. (2007) The emerging mental health strategy of the European Union: a multi-level work-in-progress. Health Policy, Epub ahead of print. doi:10.1016/j.healthpol.2007.06.005.

Macleod, J. (2007) Cannabis use and psychois: the origins and implications of an association. Advances in Psychiatric Treatment, 13, 400-411.

Macleod, J., Oakes, R., Copello, A., et al (2004) Psychological and social sequelae of cannabis and other illicit drug use by young people: a systematic review of longitudinal, general population studies. Lancet, 363, 1579-1588.

Moore, T. H. M., Zammit, S., Lingford-Hughes, A., et al (2007) Cannabis use and risk of psychotic or affective mental health outcomes: a systematic review. Lancet, 370, 319-328.

VanReekum, R., Streiner, D. L. \& Conn, D. K. (2001) Applying Bradford Hill's criteria for causation to neuropsychiatry: challenges and opportunities. Journal of Neuropsychiatry and Clinical Neurosciences, 13, 318-325. 